\title{
Efectividad de los métodos diagnósticos para la detección de ehrlichiosis monocítica humana y canina
}

\author{
Manuel Franco-Zetina', Jaime Adame-Gallegos ${ }^{2}$ y Karla Dzul-Rosado
}

${ }^{1}$ Centro de Investigaciones Regionales "Dr. Hideyo Noguchi", Universidad Autónoma de Yucatán. México.

${ }^{2}$ Campus Universitario \#2, Facultad de Ciencias Químicas,

Universidad Autónoma de Chihuahua. México.

Los autores declaran no tener conflicto de interés alguno.

Financiamiento: Este estudio fue financiado por el proyecto SALUD-2015-2-261885 otorgado a la Dra. Karla Rossanet Dzul Rosado por el Consejo Nacional de Ciencia y Tecnología (Conacyt)

México.

Recibido: 24 de noviembre de 2018

Aceptado: 19 de junio de 2019

Correspondencia a: Manuel Franco-Zetina franco.zetina1@gmail.com

\section{Effectivity of diagnostic methods for the detection of human and canine monocytic ehrlichiosis}

Ehrlichiosis is a disease transmitted by tick's bite that affect dogs and humans caused by the species Ehrlichia canis and E. chaffeensis, respectively. These bacteria are obligated intracellular gram negatives, with a cocoid to pleomorph aspect and can infect monocytes and trigger symptoms such as high fever, anorexia, thrombocytopenia, hemorrhages, anemia, and some serious problems such as splenomegaly, hepatomegaly and meningitis. There are several diagnostic tests for ehrlichiosis such as the hematological ones that evaluate the morphology of the monocytes in search of morulae; serological tests that includes the search of anti-Ehrlichia antibodies, although they might be limited due to cross reaction with other species. In other hand, the culture of Ehrlichia species is an effective method to obtain antigens and even develop indirect immunofluorescence assays (IFA). The polymerase chain reaction offers a definitive diagnosis associated to the use of genus-specific and species-specific primers, as well as its increased sensibility and specificity, compared to the others methods. Thus, in this review, we will discuss various methods applied to the diagnosis of this disease, as well as the advantages and disadvantages that these present.

Keywords: Ehrlichiosis; Ehrlichia canis; Ehrlichia chaffeensis; diagnostic methods.

Palabras clave: Ehrlichiosis; Ehrlichia canis; Ehrlichia chaffeensis; métodos diagnósticos.

\section{Introducción}

L a ehrlichiosis es una enfermedad causada por bacterias del género Ehrlichia pertenecientes a la familia Anaplasmataceae, transmitida por la picadura de garrapatas infectadas, que puede afectar a perros y humanos. Estas bacterias son gramnegativas intracelulares obligadas, de aspecto cocoide a pleomorfo, que se multiplican en vacuolas citoplasmáticas que contienen un número variable de microcolonias intra-citoplasmáticas o mórulas ${ }^{1,2}$. Las especies de Ehrlichia han perdido todos los genes de biosíntesis de lipopolisacáridos (LPS) y algunos otros para la síntesis de peptidoglucano, evitando de esta manera que se lleve a cabo una respuesta inmune efectiva para su eliminación. Por otra parte, la pérdida de peptidoglucano le proporciona flexibilidad y plasticidad a estas bacterias facilitando su circulación intravascular en los leucocitos infectados ${ }^{3,4}$.

Existen dos formas bacterianas de Ehrlichia que son las células de núcleos densos y células reticuladas (en inglés DC y RC, respectivamente) ${ }^{1,2,4}$. Estas dos variantes bacterianas desempeñan un papel importante en su ciclo de desarrollo, ya que las DC entran a la célula por endocitosis y, una vez dentro del endosoma, se transforman en formas intermedias para posteriormente madurar a
RC. Estas últimas se multiplican mediante fisión binaria durante $48 \mathrm{~h}$, generando otra vez, formas intermedias. Finalmente, ocurre una maduración nuevamente a DC, pasadas $72 \mathrm{~h}$, para después ocurrir una lisis de la célula hospedera y acaecer su liberación ${ }^{1,4}$.

Las especies E. chaffeensis y E. canis son los agentes etiológicos de la ehrlichiosis monocítica humana (sigla en inglés: HME) y ehrlichiosis monocítica canina (sigla en inglés: CME), respectivamente. Ambas bacterias tienden a parasitar los monocitos/macrófagos del hospedero formando las características mórulas en estas células $^{5,6}$. La garrapata Amblyomma americanum es el vector predominante de $E$. chaffeensis; sin embargo, se ha encontrado su presencia también en otras especies como Dermacentor variabilis, Ixodes pacificus, A. testudinarium, Haemaphysalis longicornis y $H$. yeni ${ }^{7,8}$. Por otra parte, la garrapata Rhipicephalus sanguineus es el vector principal de $E$. canis, pero también se ha encontrado en especies $D$. variabilis ${ }^{6,9}$.

Con respecto a su distribución geográfica, se ha identificado la presencia de E. canis en diferentes áreas entre las que se incluyen Venezuela, Costa Rica, Canadá, Italia, Portugal, España, México, Estados Unidos de América (E.U.A.) y Japón; así mismo, ha habido reportes de $E$. chaffeensis en Argentina, Israel, Italia, Mali, México, 
Portugal, Corea, Tailandia, E.U.A. y España ${ }^{10,11}$. Por otra parte, al sur de Brasil (Argentina, Uruguay y Chile) han habido pocos casos reportados de CME lo que puede deberse a la presencia de especies de garrapatas "templadas" que han demostrado ser vectores no competentes de E. canis, a comparación de las especies tropicales que abarcan de México a Brasil, exceptuando Río Grande del Sur, donde igual existe presencia de estos vectores no competentes ${ }^{12}$. Además, en Chile no ha habido casos publicados de HME, mientras que, en Brasil, únicamente se han reportado casos sospechosos ${ }^{13}$. Es probable que, la ausencia de casos de HME se deba a la falta de reservorios o factores climáticos como la temperatura, humedad o la vegetación ya que al igual que muchos artrópodos, las garrapatas son sensibles a los cambios climáticos, por lo que éstas deben adaptarse y sobrevivir ${ }^{14}$.

Las manifestaciones clínicas de HME comprenden signos y síntomas como fiebre, cefalea, mialgias, artralgias, malestar general, anorexia, escalofríos, leucopenia, trombocitopenia y anemia ${ }^{3,5}$. En cuanto a CME, dependerá de la fase en la que se encuentre (aguda, subclínica o crónica) aunque los síntomas comunes en los diferentes estadios son fiebre elevada, anorexia, opacidad corneal, pérdida de peso, epistaxis, vómito, diarreas, anemia y trombocitopenia $^{6,15}$. En casos graves, en ambas ehrlichiosis puede presentarse hepatomegalia, esplenomegalia, daños al sistema nervioso (meningitis), hemorragias y en el caso de los perros, una pérdida de peso significativa ${ }^{5,6}$. Para el diagnóstico de HME y CME se utilizan diferentes técnicas entre las que se incluyen las hematológicas, serológicas, aislamiento bacteriano y moleculares ${ }^{16}$.

El conocimiento sobre los aspectos de diagnóstico y sus limitaciones, permitiría potenciar su uso en casos sospechosos bajo estricto escrutinio de investigación, por lo que en esta revisión se tiene como objetivo realizar una revisión sistemática donde se discutan los métodos de diagnóstico empleados para la identificación de $E$. chaffeensis y E. canis, así como las ventajas y desventajas que éstos presentan.

\section{Diagnóstico hematológico: Evaluación de sangre periférica}

Este método diagnóstico se realiza llevando a cabo la tinción de un frotis de sangre periférica que puede ser teñido con Giemsa para buscar mórulas en los monocitos mediante microscopía. La presencia de éstas puede dar indicios de infección por E. chaffeensis o E. canis siendo un diagnóstico rápido y de bajo costo ${ }^{17-20}$. Este método presenta ciertas desventajas como lo son su baja sensibilidad, atribuido principalmente a la baja bacteriemia, implicando una mayor inversión de tiempo para la búsqueda de mórulas, lo que dificulta su identificación. Por otra parte, las mórulas se pueden observar únicamente durante la fase aguda de la enfermedad cuando la bacteria se multiplica en microcolonias intra-citoplasmáticas ${ }^{17,20-22}$.

Hamilton y cols., (2004) reportaron una sensibilidad de $38 \%$ en un total de 23 pacientes, los que incluían pacientes inmunocomprometidos e inmunocompetentes, determinando para éstos una sensibilidad de 100 y $17 \%$, respectivamente ${ }^{23}$. Por otra parte, también se observó una disminución de sensibilidad cuando el paciente se encontraba en tratamiento con doxiciclina.

Estudios recientes han demostrado la baja sensibilidad de este método diagnóstico, como el realizado por Daramola y cols., (2018), que evaluó los monocitos en un frotis de sangre seguido de una reacción de polimerasa en cadena (RPC) de tipo anidado ${ }^{19}$. Los resultados obtenidos demostraron que tres muestras fueron positivas mediante evaluación microscópica $(1,5 \%)$, mientras que por RPC, 47 muestras fueron positivas $(22,9 \%)$ incluidas en éstas, las tres muestras positivas por microscopía. Por otra parte, Happi y cols., (2018), pudo detectar en 12 muestras $(10,3 \%)$ la presencia de mórulas, mientras que, por RPC del gen $16 \mathrm{~S}$ ARNr obtuvo 42 resultados positivos $(36,2 \%)^{18}$.

Una variante de este método diagnóstico es utilizar la capa leucocitaria obtenida por centrifugación o algún otro método de concentración para la realización del frotis. Ésta ha sido utilizada para la identificación de leishmaniosis visceral, demostrando ser una opción diagnóstica para esta parasitosis; así mismo, en el caso de ehrlichiosis, los frotis realizados a partir del concentrado de leucocitos para la búsqueda de mórulas han presentado una mayor sensibilidad (66\%) comparado con el frotis de sangre periférica ( $8 \%)$; no obstante, las mórulas se han observado con mayor frecuencia en los linfocitos que en los monocitos ${ }^{24,25}$.

Dada la baja sensibilidad que se presenta en la evaluación de sangre periférica, un diagnóstico mediante este método no puede ser considerado definitivo ya que se pueden generar falsos negativos lo que resulta en una desventaja diagnóstica. Sin embargo, puede utilizarse como un método de tamizaje para posteriormente aplicar una prueba molecular y/o serológica.

\section{Serología y cultivo celular}

Las pruebas serológicas para esta enfermedad se basan en la búsqueda de anticuerpos anti-Ehrlichia utilizando métodos como el ensayo por inmunoabsorción ligado a enzimas (ELISA, por sus siglas en inglés) e inmunofluorescencia indirecta (IFI) ${ }^{26}$. Hoy en día, existen en el mercado pruebas comerciales que permiten la detección de anticuerpos IgG contra E. canis utilizando polipéptidos sintéticos de proteínas p30 y p30-1 de esta especie, como lo son las pruebas SNAP $3 \mathrm{Dx}^{\circledR}$ y SNAP $4 \mathrm{Dx}^{\circledR} \mathrm{de}$ laboratorios IDEXX $^{27-29}$. 
Harrus y cols., (2002), llevaron a cabo un estudio comparativo entre tres pruebas de ELISA comerciales para la detección de anticuerpos IgG contra E. canis: las pruebas rMAP2-ELISA, Immunocomp ${ }^{\circledR}$ (Biogal, Israel) y SNAP

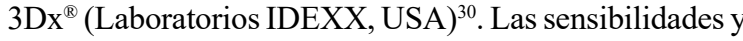
especificidades fueron de 71 y $85 \%$ para rMAP2-ELISA, 86 y $98 \%$ para Immunocomp ${ }^{\circledR}$ y 71 y $100 \%$ para SNAP $3 \mathrm{Dx}^{\circledR}$. Por otra parte, la prueba ELISA SNAP $4 \mathrm{Dx}^{\circledR}$ Plus ha demostrado ser una prueba prometedora para el diagnóstico de $E$. canis, al tener una sensibilidad y especificidad de 97,8 y $92,3 \%$, respectivamente ${ }^{31}$.

Para la detección de E. chaffeensis, Chikeka y cols., (2016), optimizaron una prueba de ELISA basada en péptidos sintéticos TRP120 y TRP32, dos proteínas inmunoreactivas de E. chaffeensis más sensibles para el serodiagnóstico $^{26}$. La prueba sólo con el péptido TRP120, presentó una sensibilidad en un rango de $88-100 \%$ y una especificidad de $71-90 \%$. Sin embargo, la prueba con los péptidos combinados (TRP120/TRP32) disminuyó la sensibilidad a un rango de $54-77 \%$ con un ligero aumento de la especificidad a un rango de 81-92\%.

Los ensayos por IFI son considerados el estándar de oro para el diagnóstico de CME y HME, pero éstos no pueden diferenciar anticuerpos contra las diferentes especies de Ehrlichia, ya que se ha reportado reactividad cruzada entre $E$. canis, E chaffeensis y E. ewingii, así como con Anaplasma phagocytophilum, pudiendo generar falsos positivos y ocasionar que el agente etiológico de la infección no sea determinado ${ }^{26,30,32}$. El valor de sensibilidad de esta prueba dependerá de las semanas transcurridas desde el inicio de la infección, teniendo valores entre 82 y $100 \%$, mientras que, la especificidad, oscila entre 67 y $100 \%$ debido a la reactividad cruzada que se presenta ${ }^{33}$. De igual manera, estos dos parámetros pueden variar con base en la composición de los antígenos utilizados, así como el formato de la prueba, ya que utilizar proteínas recombinantes, sintéticas o microorganismos completos provenientes de cultivo, puede causar diferencias en la reactividad antígeno-anticuerpo afectando los valores de sensibilidad y especificidad ${ }^{30}$.
En la Tabla 1 se muestra un resumen comparativo entre las diferentes pruebas serológicas aplicadas al diagnóstico de ehrlichiosis.

Para optimizar los parámetros de sensibilidad y especificidad de la IFI, se recurre frecuentemente al aislamiento de la especie de Ehrlichia de interés y obtener así, el antígeno a utilizar. Los cultivos de $E$. canis y $E$. chaffeensis se realizan generalmente en una línea celular de macrófagos caninos (DH82). Sin embargo, también existen otras opciones como son las células Vero (células de riñón de mono verde) y HL60 (células de leucemia promielocítica humana). No obstante, el cultivo de éstas resulta ser un proceso difícil y que requiere de experiencia ${ }^{34,35}$. Por otra parte, se ha reportado el aislamiento de $E$. canis en líneas celulares de garrapatas. Han presentado un porcentaje mayor al 50\% de células infectadas las DVE1 (Dermacentor variabilis), IDE8 y ISE6 (Ixodes scapularis) y RAE25 (Rhipicephalus appendiculatus), demostrándose de esta manera su propagación in vitro en líneas celulares de garrapatas no-vectores ${ }^{36}$.

Para el diagnóstico de CME resulta efectivo realizar pruebas comerciales, preferentemente la prueba SNAP 4Dx Plus, que consta de parámetros de sensibilidad y especificidad óptimos para su diagnóstico. La IFI resulta ser una buena opción para la detección de E. canis y $E$. chaffeensis, dado que presenta parámetros de sensibilidad y especificidad que pueden llegar a ser mayores a 90\%, los que también se pueden optimizar mediante la obtención de antígenos a partir de cultivos puros. Por otra parte, presenta la desventaja de reactividad cruzada entre los diferentes miembros de la familia Anaplasmataceae impidiendo la especiación del agente infeccioso; de igual manera, es una prueba que generalmente se realiza en laboratorios de referencia.

En Chile, el Laboratorio Biomédico del Instituto de Salud Pública es un laboratorio de referencia en el que el Sub-departamento de Enfermedades Infecciosas (Bacteriología) realiza el diagnóstico de Ehrlichia spp. en muestras clínicas mediante técnicas moleculares.

\begin{tabular}{|c|c|c|c|c|c|c|}
\hline Prueba & Especie & Sensibilidad & Especificidad & Antígeno & Formato & Referencia \\
\hline rMAP2-ELISA & E. canis & $71 \%$ & $85 \%$ & Proteína recombinante MAP2 (Major Antigenic Protein 2) & ELISA & \multirow[t]{3}{*}{30} \\
\hline Immunocomp ${ }^{\circledR}$ & E. canis & $86 \%$ & $98 \%$ & Microorganismo entero proveniente de cultivo & dot-ELISA & \\
\hline SNAP 3Dx ${ }^{\circledR}$ & E. canis & $71 \%$ & $100 \%$ & Péptidos sintéticos p30 y p30-1 & dot-ELISA & \\
\hline SNAP 4Dx ${ }^{\circledR}$ Plus & E. canis & $97,8 \%$ & $92,3 \%$ & Péptidos sintéticos p30 y p30-1 & dot-ELISA & 31 \\
\hline ELISA TRP120 & E. chaffeensis & $88-100 \%$ & $71-90 \%$ & \multirow[t]{2}{*}{ Péptidos sintéticos TRP120 y TRP32 } & \multirow[t]{2}{*}{ ELISA } & \multirow[t]{2}{*}{26} \\
\hline ELISA TRP120/TRP32 & E. chaffeensis & $54-77 \%$ & $81-92 \%$ & & & \\
\hline$|F|$ & Ehrlichia spp. & $82-100 \%$ & $67-100 \%$ & Células DH82 infectadas & Inmunofluorescencia & 33 \\
\hline
\end{tabular}




\section{Diagnóstico molecular: RPC}

Para el diagnóstico de ehrlichiosis se han desarrollado métodos género-específicos y especie-específicos mediante RPC, permitiendo optimizar el diagnóstico en fases agudas de la enfermedad.

En cuanto al diagnóstico género-específico, existen métodos basados en la amplificación de genes codificantes para tio-disulfuro oxido-reductasas (proteínas $d s b$ ) los que presentan regiones altamente conservadas entre las diversas especies de Ehrlichia ${ }^{37,38}$. Los cebadores Dsb-330 y Dsb-728 fueron diseñados basados en el alineamiento del gen de $728 \mathrm{pb}$ de la proteína $d s b$ de cinco especies de Ehrlichia (no incluida E. ewingii), que amplifica un fragmento de 409 pb demostrándose que la sensibilidad era para una sola copia del gen $d s b$ y 10 copias para E. chaffensis y E. canis, respectivamente. La especificidad de esta prueba fue de $100 \%$ con especies de Rickettsia y Anaplasma ${ }^{39}$. Por otra parte, el par de cebadores Dsb-321 y Dsb-671 reportados por Doyle y cols., (2005), fueron diseñados para la amplificación género-específica de este mismo gen mediante RPC de tiempo real obteniéndose un amplificado de $378 \mathrm{pb}^{37}$. Sin embargo, también se desarrollaron sondas TaqMan permitiendo la amplificación especie-específica de $E$. chaffeensis, E. canis y E. ewingii con una especificidad de $100 \%$ para cada una de éstas y una sensibilidad analítica de 50 copias del gen $d s b$ independientemente de la especie ${ }^{37}$.

Por otra parte, el desarrollo de métodos diagnósticos especie-específicos ha permitido determinar de manera directa el agente etiológico de la infección. Se ha desarrollado y validado una RPC cuantitativa (RPCc) en tiempo real multiplex para la detección específica de $E$. chaffeensis y A. phagocytophilum con la cual se demostró discriminación de otras especies de Ehrlichia y Anaplasma, así como otros agentes patógenos relacionados, como lo es Rickettsia spp. El gen diana amplificado fue una proteína de RPC de longitud variable (VLPT, por Variable-Length PCR Target) y $m s p 2$ (Major Surface Protein 2) para E. chaffeensis y A. phagocytophilum, respectivamente. Con respecto al ensayo multiplex, para E. chaffeensis, se obtuvo una sensibilidad de $95 \%$ y una especificidad de $99 \%$ en clínica ${ }^{40}$.

Para E. canis se han desarrollado métodos especieespecíficos entre los que destacan los que amplifican para el gen 16S ARNr y los genes codificantes de las proteínas p28 y p30 ${ }^{41-43}$. La RPC anidada del gen $16 \mathrm{~S}$ ARNr ha sido utilizada para incrementar la especificidad hacia la detección de ADN de E. canis mostrando buenos resultados. Se utilizan los cebadores ECC y ECB obteniéndose un amplificando de 478 pb para Ehrlichia spp. y especies relacionadas. El par de cebadores internos HE-3 y ECA dan como resultado un amplificado de 389 pb únicamente de $E$. canis $^{41}$. Por otra parte, las proteínas inmunodominantes p 28 y p30 se encuentran presentes en las especies de E. canis y $E$. chaffensis y son codificadas por familias multigénicas. Los amplificados para los genes de estas proteínas han demostrado tener mejor especificidad que el amplificado de 16S ARNr y en el caso de la RPC para p30, también se ha demostrado una mayor sensibilidad ${ }^{42,43}$. No obstante, el amplificado para p28 tiene una menor sensibilidad que otros ensayos de RPC para Ehrlichia spp. atribuido al gran tamaño del amplificado obtenido $(843 \mathrm{pb})^{43}$.

Además, un ensayo prometedor para el diagnóstico de ehrlichiosis ha sido el panel diagnóstico de diversos agentes patógenos transmitidos por picaduras de garrapatas desarrollado por Shen y cols., (2018), ya que este método permite no sólo el diagnóstico de $E$. chaffeensis y E. canis, sino también de otros agentes como Rickettsia spp., Anaplasma spp. e incluso Borrelia spp. mediante una RPCc, amplificando el gen 16s ARNr con un amplicón de 101 pb y una sensibilidad analítica de 10 copias por reacción. Cabe destacar que no reportaron en su estudio reacción cruzada alguno con otro tipo de bacterias patógenas de importancia veterinaria. Sin embargo, la única desventaja es que los ensayos se realizaron en muestras de animales, por lo que falta evaluar la efectividad de este método en muestras de procedencia humana ${ }^{44}$.

El empleo de técnicas de RPC ha permitido el diagnóstico de ehrlichiosis en etapas agudas y crónicas de la enfermedad y, a comparación con los métodos serológicos por IFI y ELISA, éste tiene una mejor sensibilidad y especificidad debido a la capacidad de diseñar cebadores dirigidos a un género o especie en particular.

\section{Conclusión}

La ehrlichiosis es una enfermedad cuyo diagnóstico se dificulta ya que ésta suele confundirse con otras enfermedades febriles. Si bien, la prevalencia de infecciones por Ehrlichia spp. dependerá del área geográfica, la implementación de técnicas idóneas para su diagnóstico es indispensable en áreas en donde la enfermedad tiene una mayor relevancia epidemiológica. Así mismo, el diagnóstico no debe centrarse en humanos, sino también en la población canina la que juega un papel de centinela al ser hospederos de garrapatas vectores de estas bacterias.

Todos los métodos diagnósticos constan de diferentes valores de sensibilidad y especificidad, por lo que se debe seleccionar el adecuado con base en la sintomatología y el tiempo transcurrido con la infección, ya que en el caso de la evaluación de sangre periférica y detección 
de anticuerpos IgG, el tiempo transcurrido determina su sensibilidad.

Los métodos serológicos por medio de pruebas comerciales resultan efectivos para el diagnóstico de $E$. canis siendo uno de los mejores la prueba SNAP 4Dx ${ }^{\circledR}$ Plus. Sin embargo, la IFI resulta tener valores de especificidad y sensibilidad que pueden llegar a $100 \%$ y además de ser el estándar de oro, resulta efectivo para el diagnóstico de Ehrlichia spp. en perros y humanos.

Los métodos moleculares por RPC son altamente sensibles y específicos en comparación con otros métodos diagnósticos, permitiendo la detección de la bacteria en etapas agudas y crónicas de la enfermedad. Sin embargo, para fines diagnósticos, es suficiente con determinar la presencia de $\mathrm{ADN}$ bacteriano. Así mismo, aquellos métodos que tienen bajos valores de sensibilidad y especificidad no significan que no puedan ser empleados, ya que pueden aplicarse como un método de tamizaje, es el caso de la evaluación de monocitos en sangre periférica. Por consiguiente, la implementación de métodos que impliquen la detección de Ehrlichia spp. puede ayudar a detectar de manera oportuna la infección y evitar que evolucione a cuadros fatales. Sin embargo, no debe aplicarse el diagnóstico en áreas donde estas infecciones no han sido documentadas ya que el uso de pruebas diagnósticas en forma discriminada puede llevar a un diagnóstico erróneo con consecuencias adversas.

\section{Resumen}

La ehrlichiosis es una enfermedad transmitida por la picadura de garrapatas que afecta a perros y humanos, causada por las especies Ehrlichia canis y E. chaffeensis, respectivamente. Estas bacterias son gramnegativas, intracelulares obligadas, de aspecto cocoide a pleomorfo, que infectan los monocitos y desencadenan síntomas como fiebre elevada, anorexia, trombocitopenia, hemorragias, anemia y problemas graves como esplenomegalia, hepatomegalia y meningitis. Para diagnosticar esta enfermedad existen diversos métodos, entre los que se encuentran los hematológicos que evalúan la morfología de los monocitos en búsqueda de mórulas y la serología, que incluye la búsqueda de anticuerpos anti-Ehrlichia, pero que se encuentra limitado debido a la reactividad cruzada que presenta. Por otra parte, el cultivo de especies de Ehrlichia ha resultado ser un método efectivo para la obtención de antígenos y así desarrollar ensayos por inmunofluorescencia indirecta (IFI). El método por reacción de polimerasa en cadena ofrece un diagnóstico definitivo por tener una mayor sensibilidad y especificidad que los otros métodos, al haberse desarrollado cebadores género-específicos, así como especie-específicos. En esta revisión, se discutirán los diversos métodos aplicados al diagnóstico de esta enfermedad, así como las ventajas y desventajas que estos presentan.

\section{Referencias bibliográficas}

1.- Thirumalapura N R, Walker D H. Molecular Medical Microbiology. 2nd ed. Boston: Academic Press; 2015. Chapter 109, Ehrlichia; 2011-32.

2.- McBride J W, Olano J P, Ismail N. Molecular Detection of Human Bacterial Pathogens. 1st ed. United States: CRC Press, Tylor \& Francis Group; 2011. Chapter 55, Ehrlichia; p. 648. Available from: http://books.google.com/ books? $\mathrm{id}=$ nnGhc44bypAC\&pgis $=1$.

3.- Rikihisa Y. Molecular events involved in cellular invasion by Ehrlichia chaffeensis and Anaplasma phagocytophilum. Vet Parasitol. 2010; 167 (2-4): 155-66. doi: 10.1016/j. vetpar.2009.09.017.

4.- Ismail N, McBride J W. Tick-borne emerging infections: Ehrlichiosis and Anaplasmosis. Clin Lab Med 2017; 37 (2): 317-40. Available from: http://dx.doi.org/10.1016/j.cl1.2017.01.006.

5.- Saito T, Walker D. Ehrlichioses: An important one health opportunity. Vet Sci 2016; 3 (4): 20. doi: 10.3390/vetsci3030020.

6.- Harrus S, Waner T. Diagnosis of canine monocytotropic ehrlichiosis (Ehrlichia canis): An overview. Vet J. 2011; 187 (3): 292-6.
Available from: http://dx.doi.org/10.1016/j. tvj1.2010.02.001.

7.- Ndip L M, Labruna M, Ndip R N, Walker D H, McBride J W. Molecular and clinical evidence of Ehrlichia chaffeensis infection in cameroonian patients with undifferentiated febrile illness. Ann Trop Med Parasitol 2010; 103 (8): 719-25. doi: 10.1179/000349809X12554106963753.

8.- Trout Fryxell R T, Hendricks B M, Pompo K, Mays S E, Paulsen D J, Operario D J, et al. Investigating the adult Ixodid tick populations and their associated Anaplasma, Ehrlichia, and Rickettsia bacteria at a Rocky Mountain Spotted Fever hotspot in Western Tennessee. Vector-Borne Zoonotic Dis 2017; 17 (8): 52738. Available from: http://online.liebertpub. com/doi/10.1089/vbz.2016.2091.

9.- Sosa-Gutiérrez C G, Quintero-Martínez T, Vargas-Sandoval M, Gordillo-Pérez G. Primer análisis filogenético de Ehrlichia canis en perros y garrapatas de México. Estudio preliminar. Rev MVZ Cordoba 2016; 21 (3): 5569-76. doi:10.21897/rmvz.831.

10.- André M R. Diversity of Anaplasma and Ehrlichia/Neoehrlichia agents in terrestrial wild carnivores worldwide: implications for human and domestic animal health and wildlife conservation. Front Vet Sci 2018; 5: 293. doi: 10.3389/fvets.2018.00293.

11.- Paddock C D, Childs JE. Ehrlichia chaffeensis: a prototypical emerging pathogen. Clin Microbiol Rev 2003;16 (1): 37-64. doi: $10.1128 / \mathrm{cmr} \cdot 16.1 .37-64.2003$

12.- Moraes-Filho J, Krawczak F S, Costa F B, Soares J F, Labruna M B. Comparative evaluation of the vector competence of four South American populations of the Rhipicephalus sanguineus group for the bacterium Ehrlichia canis, the agent of canine monocytic ehrlichiosis. PLoS One 2015; 10 (9): 1-16. https://doi.org/10.1371/journal. pone. 0139386.

13.- Calic S B, Galvão M A M, Bacellar F, Rocha C M B M, Mafra C L, Leite R C, et al. Human ehrlichioses in Brazil: first suspect cases. Braz J Infect Dis 2005; 8 (3): 7-9. doi: /S141386702004000300011.

14.- Estrada-Peña A, Ayllón N, de la Fuente J. Impact of climate trends on tick-borne pathogen transmission. Front Physiol 2012;3 (64). doi: 10.3389/fphys.2012.00064.

15.- Das M, Konar S. Clinical and hematological study of canine ehrlichiosis with other hemoprotozoan parasites in Kolkata, West Bengal, India. Asian Pac J Trop Biomed 
2013; 3 (11): 913-5. doi: 10.1016/S22211691(13)60178-1.

16.- Botelho-Nevers E. Rickettsiosis y ehrlichiosis. EMC - Dermatología 2014; 48 (3): 1-10. Available from: http://linkinghub.elsevier.com/ retrieve/pii/S1761289614684041.

17.- Derakhshandeh N, Sharifiyazdi H, Hasiri M A. Molecular detection of Ehrlichia spp. in blood samples of dogs in southern Iran using polymerase chain reaction Vet Res Forum. 2017; 8 (4): 347-51. PMID: 29326795.

18.- Happi A N, Toepp A J, Ugwu C A, Petersen C A, Sykes J E. Detection and identification of blood-borne infections in dogs in Nigeria using light microscopy and the polymerase chain reaction. Vet Parasitol Reg Stud Reports. 2018; 11(September 2017): 55-60. https://doi. org/10.1016/j.vprsr.2017.12.002.

19.- Daramola O O, Takeet M I, Oyewusi I K, Oyekunle M A, Talabi A O. Detection and molecular characterisation of Ehrlichia canis in naturally infected dogs in South West Nigeria. Acta Vet Hung 2018; 66 (1): 85-95. Available from: http://www.ncbi.nlm.nih.gov/ pubmed/29580089\%0Ahttp://www.akademiai. com/doi/10.1556/004.2018.008.

20.- Ybañez R H D, Ybañez A P, Arnado L L A, Belarmino L M P, Malingin K G F, Cabilete P B C, et al. Detection of Ehrlichia, Anaplasma, and Babesia spp. in dogs in Cebu, Philippines. Vet World 2018; 11 (1): 14-9. doi: 10.14202/ vetworld.2018.14-19.

21.- Badillo-Viloria, María, Díaz-Pérez, Anderson, Orozco-Sánchez, Christian, Lavalle-Galvis R. Infection by Ehrlichia canis and Anaplasma sp. in dogs attended in veterinary clinics, Barranquilla, Colombia. Rev MVZ Cordoba. 2017; 22 (Suplemento): 6023-33. https://doi. org/10.21897/rmvz.1072.

22.- Bai L, Goel P, Jhambh R, Kumar P, Joshi V G. Molecular prevalence and haemato-biochemical profile of canine monocytic ehrlichiosis in dogs in and around Hisar, Haryana, India. J Parasit Dis 2017; 41 (3): 647-54. doi: 10.1007/s12639016-0860-8.

23.- Hamilton K S, Standaert S M, Kinney M C. Characteristic peripheral blood findings in human ehrlichiosis. Mod Pathol 2004; 17 (5): 512-7. doi: 10.1038/modpathol.3800075.

24.- Mylonakis M E, Koutinas A F, Billinis C, Leontides L S, Kontos V, Papadopoulos O, et al. Evaluation of cytology in the diagnosis of acute canine monocytic ehrlichiosis (Ehrlichia canis): A comparison between five methods. Vet Microbiol 2003; 91 (2-3): 197-204. PMID: 12458168.

25.- Salam M A, Khan M G M, Bhaskar K R H, Afrad M H, Huda M M, Mondal D. Peripheral blood buffy coat smear: A promising tool for diagnosis of visceral leishmaniasis. J Clin Microbiol 2012; 50 (3): 837-40. doi: 10.1128/ JCM.05067-11.
26.- Chikeka I, Matute A J, Dumler J S, Woods C W, Mayorga O, Reller M E, et al. Use of peptide ELISA followed by IFA to document Ehrlichia chaffeensis as a cause of febrile illness in Nicaragua. J Clin Microbiol 2016; 54 (April): 1-21. doi: 10.1128/JCM.03331-15.

27.- Suh G H, Ahn K S, Ahn J H, Kim H J, Leutenegger C, Shin SS. Serological and molecular prevalence of canine vector-borne diseases (CVBDs) in Korea. Parasit Vectors. 2017; 10(1): 1-8. doi: 10.1186/s13071-017-2076-x.

28.- Beall M J, Alleman A R, Breitschwerdt E B, Cohn LA, Couto CG, Dryden MW, et al. Seroprevalence of Ehrlichia canis, Ehrlichia chaffeensis and Ehrlichia ewingii in dogs in North America. Parasit Vectors. 2012;5(1):1-11. doi: 10.1186/1756-3305-5-29

29.- Krämer F, Schaper R, Schunack B, Połozowski A, Piekarska J, Szwedko A, et al. Serological detection of Anaplasma phagocytophilum, Borrelia burgdorferi sensu lato and Ehrlichia canis antibodies and Dirofilaria immitis antigen in a countrywide survey in dogs in Poland. Parasitol Res. 2014; 113 (9): 3229-39. doi: 10.1007/s00436-014-3985-7.

30.- Harrus S, Alleman A R, Bark H, Mahan S $\mathrm{M}$, Waner T. Comparison of three enzymelinked immunosorbant assays with the indirect immunofluorescent antibody test for the diagnosis of canine infection with Ehrlichia canis. Vet Microbiol 2002; 86 (4): 361-8. PMID: 11955786.

31.- Piantedosi D, Neola B, D’Alessio N, Di Prisco F, Santoro M, Pacifico L, et al. Seroprevalence and risk factors associated with Ehrlichia canis, Anaplasma spp., Borrelia burgdorferi sensu lato, and D. immitis in hunting dogs from southern Italy. Parasitol Res 2017; 116 (10): 2651-60. doi: 10.1007/s00436-017-5574-z

32.- Koh F X, Kho K L, Kisomi M G, Wong L P, Bulgiba A, Tan P E, et al. Ehrlichia and Anaplasma infections: Serological evidence and tick surveillance in peninsular Malaysia. J Med Entomol. 2018; 55 (2): 269-76. doi: 10.1093/ jme/tjx204.

33.- Martínez-Vega P P, Bolio-González M E, Rodríguez-Vivas R I, Gutierrez-Blanco E, Pérez-Osorio C, Villegas-Pérez S L, et al. Associated factors to seroprevalence of Ehrlichia spp. in dogs of Quintana Roo, Mexico. J Trop Med 2016; 2016: 19-21. doi: 10.1155/2016/4109467.

34.- Eedunuri V K, Zhang Y, Cheng C, Chen L, Liu H, Omsland A, et al. Protein and DNA synthesis demonstrated in cell-free Ehrlichia chaffeensis organisms in axenic medium. Sci Rep] 2018; 8 (1): 9293. Available from: http:// www.nature.com/articles/s41598-018-27574-z.

35.- Kondethimmanahalli C, Ganta R. Impact of three different mutations in Ehrlichia chaffeensis in altering the global gene expression patterns. Sci Rep 2018; 8 (1): 1-13.
Available from: http://dx.doi.org/10.1038/ s41598-018-24471-3.

36.- Ferrolho J, Simpson J, Hawes P, Zweygarth E, Bell-Sakyi L. Growth of Ehrlichia canis, the causative agent of canine monocytic ehrlichiosis, in vector and non-vector ixodid tick cell lines. Ticks Tick Borne Dis 2016; 7 (4): 631-7. doi: 10.1016/j.ttbdis.2016.01.013.

37.- Doyle C K, Labruna M B, Breitschwerdt E B, Tang Y W, Corstvet R E, Hegarty B C, et al. Detection of medically important Ehrlichia by quantitative multicolor TaqMan real-time polymerase chain reaction of the $d s b$ gene. J Mol Diagnostics. 2005; 7 (4): 504-10. doi: 10.1016/S1525-1578(10)60581-8.

38.- Aguiar D M, Ziliani T F, Zhang X, Melo A L, Braga I A, Witter R, et al. A novel Ehrlichia genotype strain distinguished by the TRP36 gene naturally infects cattle in Brazil and causes clinical manifestations associated with ehrlichiosis. Ticks Tick Borne Dis 2014; 1-8. Available from: http://dx.doi.org/10.1016/j. ttbdis.2014.03.010.

39.- Labruna M B, McBride J W, Camargo L M A, Aguiar D M, Yabsley M J, Davidson W R, et al. A preliminary investigation of Ehrlichia species in ticks, humans, dogs, and capybaras from Brazil. Vet Parasitol 2007; 143 (2): 189-95. doi:10.1016/j.vetpar.2006.08.005.

40.- Reller M E, Dumler J S. Development and clinical validation of a multiplex real-time quantitative PCR assay for human infection by Anaplasma phagocytophilum and Ehrlichia chaffeensis. Trop. Med. Infect. Dis. 2018; 3 (1): 14. doi: 10.3390/tropicalmed3010014.

41.- Wen B, Rikihisa Y, Mott J M, Greene R, Kim H Y, Zhi N, et al. Comparison of nested PCR with immunofluorescent-antibody assay for detection of Ehrlichia canis infection in dogs treated with doxycycline. J Clin Microbiol. 1997; 35 (7): 1852-6. PMID: 9196207.

42.- Stich R W, Rikihisa Y, Ewing S A, Needham G R, Grover D L, Jittapalapong S. Detection of Ehrlichia canis in canine carrier blood and in individual experimentally infected ticks with a $p 30$-based PCR assay. J Clin Microbiol 2002; 40 (2): 540-6. doi: 10.1128/jcm.40.2.540546.2002 .

43.- Nakaghi A C H, Machado R Z, Ferro J A, Labruna M B, Chryssafidis A L, André M R, et al. Sensitivity evaluation of a single-step PCR assay using Ehrlichia canis p28 gene as a target and its application in diagnosis of canine ehrlichiosis. Rev Bras Parasitol Vet 2010; 19 (2): 75-9. http://dx.doi.org/10.4322/ rbpv.01902001.

44. Shen Z, Zhang M Z, Stich R W, Mitchell W J, Zhang S. Development of a tick-borne pathogen QPCR panel for detection of Anaplasma, Ehrlichia, Rickettsia, and Lyme disease Borrelia in animals. J Microbiol Methods 2018; 151: 83-9. doi: 10.1016/j.mimet.2018.05.019. 